\title{
The Quantum Hall Effect for Electrons in a Random Potential
}

\author{
H. Kunz
}

Institut de Physique Théorique, Ecole Polytechnique Fédérale de Lausanne, PHB-Ecublens, CH-1015 Lausanne, Switzerland

\section{Dedicated to Walter Thirring on his $60^{\text {th }}$ birthday}

\begin{abstract}
We assume the existence of sufficiently localised states, near the edges of each Landau band. We then prove that the Hall conductivity is quantised and the parallel conductivity vanishes, when the filling factor stays close to an integer. The Hall integer is a topological invariant, given by the Landau band index.

We also prove that at weak disorder, the localisation length diverges in each Landau band.
\end{abstract}

\section{Introduction}

Since the experimental discovery of the integer quantum Hall effect, by von Klitzing [1] in a system of electrons confined to move in a plane, there have been many theoretical attempts to explain it on a microscopical basis.

The most famous one has been that of Laughlin [2], who, on the basis of a model independent argument, concluded that the quantisation should hold quite generally, when the electronic chemical potential lies in a gap or in the region of localised states. Later on, Halperin [3] showed that Laughlin's argument leads to the conclusion that the localisation length should diverge at least for some energies, when a strong magnetic field is present, in contrast to what is presently believed to occur in the absence of a magnetic field, for a two-dimensional system.

The first proof of Laughlin's conjecture in a model was made by Thouless et al. [4], (TKNN), who considered a system of non-interacting electrons in a periodic potential and a "rational" magnetic field, the electronic chemical potential lying in a gap. Avron et al. [5] soon proved that the TKNN integers associated to the Hall conductivity had a topological interpretation. (To a certain extent the topological aspect of the problem was anticipated in the not so well-known work of Dubrovin and Novikov [6], and Novikov [7] who considered mostly the case of a periodic magnetic field.) Later on it was realised by a number of authors: Niu and Thouless $[8,9]$, Avron and Seiler [10, 11], Tao and Haldane [12], Grümm et al. [13] that the analysis of TKNN did not really need the consideration of non-interacting electrons, but could be applied to any finite system of electrons, confined to move 
on a torus. The Hall conductivity is quantised as long as the ground state is nondegenerate, and separated by a gap (in a strong sense), from the excited states. Working with such a generality, it has not been possible however, either to show that the integers correspond to the values actually observed, nor to prove that they do not change when one varies a little the magnetic field or the density (this is needed to explain the observed plateaux). Concerning this last point, however, there has always been qualitative arguments suggesting that the plateaux owe their existence to the presence of localised states due to disorder in the system.

In this work, we reconsider this problem in the case of an infinite system of noninteracting electrons, under the influence of a random potential and a magnetic field.

If the potential is weak enough, the spectrum is made of bands, called Landau bands. Assuming that all states are sufficiently localised near the edge of each of these Landau bands, we prove that the Hall conductivity $\sigma_{x y}=\frac{e^{2}}{h} n(n=0,1,2, \ldots)$ and the parallel conductivity $\sigma_{y y}=0$, when the electronic density $\varrho$ stays close to the value $\varrho=\frac{e B}{h c} n$. The conductivities here considered, are those of an infinite system (where the distinction between localised and extended states can be neatly made), and are defined by means of appropriate Kubo formulas. It may be worth noting that in order to set such formulas, the electric field has been applied adiabatically on the system. It has been shown by G. Zumbach and the author [14], that at least for some models, a static electric field does not give a quantised Hall conductivity.

Finally, our proofs also lead to the conclusion that in each Landau band, the localisation length must diverge at some energies, in agreement with Halperin's argument.

The general strategy of our proof is quite simple. Taking first the chemical potential in a gap, we show that it is possible to approximate the conductivity by that of a system in a periodic potential, with sufficiently large period. For such a system, the Hall conductivity is proved to be quantised, in essentially the same way as in the TKNN case. (We just need an extension to degenerate bands.) The integer then corresponds to a topological invariant. The actual value of the integer is computed, by using the continuity with respect to the potential.

Finally, the contribution to the Hall conductivity coming from localised states is carefully analysed. It appears that localised states may contribute to the Hall conductivity if they are degenerate, but not to the parallel conductivity. This is due to the absence of time reversal symmetry in the hamiltonian. Assuming therefore the non-degeneracy of localised states, we show that the conductivity is the same as in the case of a chemical potential in a gap. This is the expected mechanism for the existence of plateaux for the Hall conductivity as a function of the density.

We finally comment on the possible extension of these results to larger values of the potential.

\section{The Model and the Main Result}

We consider a system made of an infinite number of independent electrons of charge $e$, mass $m$, moving in the $(x, y)$ plane under the influence of a magnetic field $B$ 
applied in the $z$ direction and a random potential, due to impurities. The chemical potential, $\mu$, of the electrons is fixed, and the temperature taken to be zero. Choosing the cyclotronic energy $\frac{e B}{m c}$ and the magnetic length $\left(\frac{\hbar c}{e B}\right)^{1 / 2}$, as units of energy and length respectively, the one electron hamiltonian reads:

$$
H=\frac{1}{2} v_{x}^{2}+\frac{1}{2} v_{y}^{2}+V(x, y),
$$

where the velocity operator $\mathbf{v}$ is given by

in the Landau gauge.

$$
v_{x}=\frac{1}{i} \partial_{x}+y, \quad v_{y}=\frac{1}{i} \partial_{y}
$$

Concerning the random potential $V$, we will simply assume that its probability distribution is translation invariant and that it is bounded:

$$
\|V\|=\sup _{x, y}|V(x, y)|<\infty .
$$

Suppose we apply adiabatically an electric field in the $y$ direction on the system. A current in the $x$ and $y$ direction is generated. The corresponding conductivities can be defined by an extension of the usual Kubo formulas, to infinite systems,

$$
\begin{gathered}
\sigma_{x y}=\lim _{\varepsilon \downarrow 0} \frac{1}{i \varepsilon} \int_{0}^{+\infty} e^{-\varepsilon t} M\left(f\left[v_{x}, v_{y}(-t)\right] f\right) d t, \\
\sigma_{y y}=\lim _{\varepsilon \downarrow 0} \frac{1}{i \varepsilon} \int_{0}^{+\infty} e^{-\varepsilon t} M\left(f\left[v_{y}, v_{y}(-t)\right] f\right) d t+\frac{1}{\varepsilon} M(F),
\end{gathered}
$$

in units of $\frac{e^{2}}{\hbar} \cdot \varepsilon$ is the adiabatic switching parameter and $f$, the Fermi projector

$$
f(H)=\left\{\begin{array}{lll}
\mathbb{1} & \text { if } \quad H<\mu \\
0 & \text { if } \quad H \geqq \mu
\end{array}\right.
$$

or $f=E_{H}(-\infty, \mu)$. The notation $M(A)$ for an operator $A$ of kernel $A\left(r, r^{\prime}\right)$ means

$$
M(A)=\overline{A(r, r)},
$$

the bar denoting an average over the disorder. The electronic density $\varrho$ is given by

$$
\varrho(\mu)=M(f) \text {. }
$$

It is well known [15] that the spectrum of the hamiltonian

$$
H_{0}=\frac{1}{2} v_{x}^{2}+\frac{1}{2} v_{y}^{2}
$$

is made of eigenvalues only $\left\{n+\frac{1}{2}, n=0,1,2, \ldots\right\}$, infinitely degenerate called Landau levels. When the disorder is small enough, i.e. $\|V\|<\frac{1}{2}$, it can be proved [23] that the spectrum of the hamiltonian $H$ is contained in the intervals $A_{n}$ $=\left[n+\frac{1}{2}-\|V\|, n+\frac{1}{2}+\|V\|\right]$. Moreover under rather general conditions on the probability distribution of the potential, we expect that these intervals will be filled by the spectrum of $H$, with probability one. Therefore we will have $\sigma(H)=\bigcup_{n=0}^{\infty} A_{n}$. 
We expect also, and this will be our main assumption, that states close to the edges of these bands will be localised, probably exponentially.

Let us formulate more precisely this hypothesis.

Let

$$
\begin{aligned}
& A_{n}^{+}=\left[n+\frac{1}{2}+\|V\|-\delta_{n}, n+\frac{1}{2}+\|V\|\right], \\
& A_{n}^{-}=\left[n+\frac{1}{2}-\|V\|, n+\frac{1}{2}-\|V\|+\delta_{n}\right],
\end{aligned}
$$

with

$$
0<\delta_{n}<\|V\|
$$

The hypothesis $H$ is that, with probability one,

$\mathrm{H}_{1}$ ) The spectrum of $H$ in $A_{n}^{ \pm}$is pure point for all $n \geqq 0$ and simple.

$\mathrm{H}_{2}$ ) The localisation length is finite.

More precisely, if $\varphi_{\lambda}(r)$ is the unique eigenvector corresponding to the eigenvalue $\lambda$, the following quantity is finite, for all $n \geqq 0$,

$$
K\left(A_{n}^{ \pm}\right)=\int d^{2} r r^{2} \overline{\sum_{\lambda \in A_{n}^{ \pm}}\left|\varphi_{\lambda}(0)\right|^{2}\left|\varphi_{\lambda}(r)\right|^{2}} .
$$

These assumptions correspond to the current physical expectation, and have been proven rigorously in some lattice models without a magnetic field, [16-18], using basic results of Fröhlich and Spencer [19], and we may expect them to be rigorously proven for some continuous model with a magnetic field, like that discussed in [20], with similar techniques.

Using these assumptions, it is then possible to prove the quantisation of the Hall conductivity $\sigma_{x y}$ and the existence of plateaux, when this quantity is plotted as a function of the electronic density $\varrho$. Moreover, the result leads to the conclusion already reached by Halperin [3] on the basis of Laughlin's argument, that the localisation length must diverge in each Landau band. Whether this corresponds to truly extended states (continuous spectrum) or simply a divergence of the localisation length in the middle of the band, i.e. when $e=n+\frac{1}{2}$, remains open. The results are summarised in the following

Theorem. a) Let hypothesis $H_{1}$ and $H_{2}$ hold, then for each integer $n \geqq 0$, there exist densities

$$
\begin{aligned}
& \varrho_{-, n}=\varrho\left(n+\frac{1}{2}+\|V\|-\delta_{n}\right), \\
& \varrho_{+, n}=\varrho\left(n+\frac{3}{2}-\|V\|+\delta_{n+1}\right),
\end{aligned}
$$

such that if the electronic density $\varrho$ is in the interval $\varrho \in\left[\varrho_{-, n}, \varrho_{+, n}\right]$, then

$$
\sigma_{x y}=\frac{1}{2 \pi}(n+1), \quad \sigma_{y y}=0,
$$

$n$ is the Landau band index. It is also a topological invariant. Moreover $\frac{n+1}{2 \pi} \in\left[\varrho_{-, n}, \varrho_{+, n}\right]$.

b)

$$
K\left(A_{n}\right)=\infty
$$

for all $n \geqq 0$. 
The result (1.14) has the following interpretation. It is expected that when the energy $e$ is, in the region of exponentially localised states,

$$
\overline{\sum_{\lambda} \delta(e-\lambda)\left|\varphi_{\lambda}(0)\right|^{2}\left|\varphi_{\lambda}(r)\right|^{2}}
$$

decreases as $\exp -\frac{|r|}{\xi(e)}, \xi(e)$ being the localisation length. Equation (1.14) says that in each Landau band $A_{n}$, there must exist at least one energy $e_{c}(n)$ such that $\xi\left(e_{c}(n)\right)=\infty$. If we imagine a power law divergence $\xi(e) \sim\left|e_{c}-e\right|^{-v}$, then (1.14) says $v>\frac{1}{4}$. Present numerical work [21] suggest that $e_{c}(n)=n+\frac{1}{2}$ and $v \simeq 1$.

Finally, we note that a condition of weak disorder or strong enough magnetic field is needed for the quantisation to hold, since otherwise all states would be exponentially localised and as our results show, we would have $\sigma_{x y}=0$. The condition $\|V\|<\frac{1}{2}$ maintaining gaps in the system is too strong, and we expect the result to hold even when this condition is violated and the chemical potential lays in a region of localised states. In the conclusion, we present plausible arguments in support of this behaviour.

\section{Definitions and Technical Tools}

In the formal expression we have given for the conductivity, operators like the velocities which are unbounded appear. Our first task is to give proper meaning to such formulas, but in the process of doing this, we will introduce technical tools which will prove useful in the sequel.

Let $A_{V}$ be an operator, possibly dependent on the random potential $V . A_{V}$ will be said translation invariant, if

$$
T(a) A_{V} T(a)^{-1}=A_{V_{a}},
$$

where

$$
V_{a}(x, y)=V\left(x+a_{x}, y+a_{y}\right)
$$

and

$$
T(a)=\exp i a_{y} x \cdot \exp i\left(a_{x} p_{x}+a_{y} p_{y}\right)
$$

is the magnetic translation operator, in the Landau gauge. Examples of such operators are the velocities $v_{x}, v_{y}$ and the hamiltonian $H_{V}$.

An operator will be said to be averageable, $A_{V} \in \mathfrak{M}$ if:

1) $A_{V}$ is translation invariant.

2) $A_{V}$ possesses a kernel $A_{V}\left(r, r^{\prime}\right)$, continuous in $\left(r, r^{\prime}\right)$ on $R^{2} \times R^{2}$.

$$
\overline{\left|A_{V}(0,0)\right|}<\infty \text {. }
$$

For such operators, we can define an average

$$
M(A)=\overline{A_{V}(0,0)}=\overline{A_{V}(r, r)}, \quad \forall r
$$

because, their translation invariance implies that their kernel satisfies

$$
A_{V_{a}}\left(r, r^{\prime}\right)=e^{i a_{y}\left(x-x^{\prime}\right)} A_{V}\left(r+a, r^{\prime}+a\right) .
$$


Such operators are self-averaging, in the sense that, for an ergodic probability distribution of the potential

$$
\lim _{\Lambda \uparrow R^{2}} \frac{1}{|\Lambda|} \int_{\Lambda} d^{2} r A_{V}(r, r)=M(A)
$$

with probability one.

It is useful to consider the following class of operators $A_{V} \in \mathscr{C}$, if

1) $A_{V}$ is translation invariant;

2) $A_{V}$ possesses a kernel $A_{V}\left(r, r^{\prime}\right)$, such that

$$
|A|=\sup _{\substack{V, r \\\|V\| \text { fixed }}}\left(\int\left|A_{V}\left(r, r^{\prime}\right)\right|^{2} d r^{\prime}\right)^{1 / 2}<\infty ;
$$

3)

$$
\lim _{r \rightarrow s}\left|A_{V}\right|_{r, s}=0
$$

where

$$
\left|A_{V}\right|_{r, s}=\left(\int\left|A_{V}(r, t)-A_{V}(s, t)\right|^{2} d t\right)^{1 / 2} .
$$

These operators are essentially operators of the Carleman type, except for the supplementary continuities property 3). We recall [22] that an operator $T$ is Carleman if $\forall f \in D(T), \exists k(r)$, such that

$$
|(T f)(r)| \leqq\|f\| k(r) \quad \text { a.e. }
$$

This is equivalent [22] to the fact that $T$ possesses a measurable kernel $T\left(r, r^{\prime}\right)$ such that

$$
|T|(r)=\left(\int\left|T\left(r, r^{\prime}\right)\right|^{2} d r^{\prime}\right)^{1 / 2}<\infty .
$$

Moreover, if $S$ is bounded, $T S$ is Carleman and

$$
|T B|(r) \leqq\|B\||T|(r) .
$$

Let us design by $\mathfrak{B}$ the class of bounded translation invariant operators $B_{V}$, with

$$
\|B\|=\sup _{V,\|V\| \text { fixed }}\left\|B_{V}\right\|<\infty
$$

Then we have

Proposition 1. If $A \in \mathscr{C}, B \in \mathfrak{B}$, then $A B \in \mathscr{C}$.

It is clear that property 1) and 2) are satisfied for $A B$, since

$$
|A B| \leqq|A|\|B\| \text {. }
$$

On the other hand

$$
|A B|_{r, s}^{2}=\sup _{\substack{\varphi \\\|\varphi\|=1}}\left|\int[A(r, t)-A(s, t)]\left(B \varphi^{*}\right)(t) d t\right|,
$$

hence

$$
|A B|_{r, s} \leqq|A|_{r, s}\|B\|
$$


The following property shows the usefulness of the introduction of the class $\mathscr{C}$ of operators.

Proposition 2. If $A \in \mathscr{C}$ and $B^{+} \in \mathscr{C}$, then $A B \in \mathfrak{M}$.

The result follows simply, from Schwartz inequality which gives $|A B| \leqq|A|\left|B^{+}\right|$ and

$$
\left|(A B)(r, s)-(A B)\left(r^{\prime}, s^{\prime}\right)\right| \leqq|A|_{r, r^{\prime}}\left|B^{+}\right|+\left|B^{+}\right|_{s, s^{\prime}}|A| .
$$

We now have to prove that a number of operators which will appear later on belong to the various classes we have introduced.

Consider first the hamiltonian

$$
H_{0}=\frac{1}{2} v_{x}^{2}+\frac{1}{2} v_{y}^{2} .
$$

Defining the creation and annihilation operators

$$
a=\frac{1}{\sqrt{2}}\left(v_{x}+i v_{y}\right), \quad a^{+}=\frac{1}{\sqrt{2}}\left(v_{x}-i v_{y}\right),
$$

satisfying the commutation relation

$$
\left[a, a^{+}\right]=1
$$

we see that

$$
H_{0}=a^{+} a+\frac{1}{2}
$$

so that the spectrum of $H_{0}, \sigma\left(H_{0}\right)$ is that of an harmonic oscillator

$$
\sigma\left(H_{0}\right)=\left\{n+\frac{1}{2}, n=0,1, \ldots\right\} \text {. }
$$

The corresponding projectors $P_{n}$, are given in the Landau gauge, where $v_{x}=p_{x}+y$, $v_{y}=p_{y}$, by operators of kernel

$$
P_{n}\left(x y ; x^{\prime} y^{\prime}\right)=\frac{1}{2 \pi} \int_{-\infty}^{+\infty} d k e^{i k\left(x-x^{\prime}\right)} \varphi_{n}(y+k) \varphi_{n}\left(y^{\prime}+k\right)
$$

where

$$
\varphi_{n}(y)=\frac{}{\pi^{1 / 4}\left(2^{n} n !\right)^{1 / 2}} H_{n}(y) e^{-\frac{y^{2}}{2}},
$$

$H_{n}(y)$ being the hermite polynomial of order $n$.

We have

Proposition 3. $G_{z}^{0}=\left(z-H_{0}\right)^{-1} \in \mathscr{C}$ if $z \notin \sigma\left(H_{0}\right)$.

Proof. $G_{z}^{0}$ is translation invariant and a Carleman operator. Indeed, its kernel $G_{z}^{0}\left(r, r^{\prime}\right)$ is such that

$$
\left|G_{z}^{0}\right|^{2}(r)=\int\left|G_{z}^{0}\left(r, r^{\prime}\right)\right|^{2} d r^{\prime}=\left(G_{z}^{0} G_{z^{*}}^{0}\right)(r, r)=\sum_{n=0}^{\infty} \frac{P_{n}(r, r)}{\left|z-n-\frac{1}{2}\right|^{2}} .
$$


From (2.17) it follows that $P_{n}(r, r)=\frac{1}{2 \pi}$, and we have $\left|G_{z}^{0}\right|<\infty$. On the other hand

$$
\left|G_{z}^{0}\right|_{r, s}^{2}=\left(G_{z}^{0} G_{z^{*}}^{0}\right)(r, r)+\left(G_{z}^{0} G_{z^{*}}^{0}\right)(s, s)-\left(G_{z}^{0} G_{z^{*}}^{0}\right)(s, r)-\left(G_{z}^{0} G_{z^{*}}^{0}\right)(r, s),
$$

so that

$$
\left|G_{z}^{0}\right|_{r, s}^{2}=\sum_{n=0}^{\infty} \frac{1}{\left|z-n-\frac{1}{2}\right|^{2}}\left[P_{n}(r, r)+P_{n}(s, s)-P_{n}(s, r)-P_{n}(r, s)\right],
$$

and since from $(2.17)$ and (2.18) it follows that $\left|P_{n}(r, s)\right| \leqq \frac{1}{2 \pi}$ and $P_{n}(r, s)$ is continuous on $R^{2} \times R^{2}$, we have by dominated convergence

$$
\lim _{r \rightarrow s}\left|G_{z}^{0}\right|_{r, s}=0 .
$$

We have also

Proposition 4. $v_{x} G_{z}^{0} \in \mathfrak{B}$ if $z \notin \sigma\left(H_{0}\right)$.

Proof. We have

$$
a^{+} a P_{n}=n P_{n},
$$

therefore

$$
\left\|a G_{z}^{0}\right\|^{2}=\sup _{\varphi:\|\varphi\|=1} \sum_{n=0}^{\infty} \frac{n}{\left|z-n-\frac{1}{2}\right|^{2}}\left(\varphi, P_{n} \varphi\right)<\infty .
$$

Similarly

$$
\left\|a^{+} G_{z}^{0}\right\|^{2}=\sup _{\varphi:\|\varphi\|=1} \sum_{n=0}^{\infty} \frac{n+1}{\left|z-n-\frac{1}{2}\right|^{2}}\left(\varphi, P_{n} \varphi\right)<\infty .
$$

On the other hand we have

Proposition 5. $G_{z}=(z-H)^{-1} \in \mathscr{C} \cap \mathfrak{B}, \underset{y}{v_{x}} G_{z} \in \mathfrak{B}$, if

$$
\inf _{V:\|\boldsymbol{V}\| \text { fixed }}\left\{\operatorname{dist}(z, \sigma(H)), \operatorname{dist}\left(z, \sigma\left(H_{0}\right)\right)\right\}>0 .
$$

Proof. The second resolvent equation gives

$$
\begin{gathered}
G_{z}=G_{z}^{0}+G_{z}^{0} V G_{z}, \\
v_{x} G_{z}=v_{x} G_{z}^{0}+v_{x} G_{z}^{0} V G_{z} .
\end{gathered}
$$

Hence from Proposition 1, it follows that

$$
\left|G_{z}\right| \leqq\left|G_{z}^{0}\right|+\left|G_{z}^{0}\right|\|V\|\left\|G_{z}\right\|,
$$

and therefore

$$
G_{z} \in \mathscr{C} \cap \mathfrak{B}
$$

Similarly, the second equation gives $v_{x} G_{z} \in \mathfrak{B}$. Let $f$ be the spectral projector $E(-\infty, \mu)$. Then we have 
Proposition 6. $f \in \mathscr{C} \cap \mathfrak{B}, f v_{x}(t) \in \mathscr{C} \cap \mathfrak{B}, \forall t \in R$.

Proof. Writing $f=G_{i}(i-H) f$, we see from Propositions 1 and 5, that $f \in \mathscr{C} \cap \mathfrak{B}$. Writing $f v_{x}(t)=e^{i t H}(i-H) f G_{i} v_{x} e^{-i t H}$, we see that $f v_{x}(t) \in \mathfrak{B}$. Finally writing $f v_{x}(t)=f f v_{x}(t)$, we see from Proposition 1 , that $f v_{x}(t) \in \mathscr{C} \cap \mathfrak{B}$.

Finally, we note that the operation $M(A)$ has all the properties of a trace. We have, indeed

\section{Proposition 7.}

1) $M^{*}(A)=M\left(A^{+}\right)$if $A, A^{+} \in \mathfrak{M}$.

2) $M\left(A A^{+}\right) \geqq 0$ if $A \in \mathscr{C}$.

3) $|M(A B)| \leqq M\left(A A^{+}\right)^{1 / 2} M\left(B^{+} B\right)^{1 / 2}$ if $\left(A, B^{+}\right) \in \mathscr{C}$.

4) $M(A B)=M(B A)$ if $\left(A, A^{+}, B, B^{+}\right) \in \mathscr{C}$.

Proof. 1) and 2) are evident from Proposition 2.

3 ) is simply Schwartz inequality following from 2).

4) is proved as follows:

$$
M(A B)=\overline{\int d r A_{V}(0, r) B_{V}(r, 0)},
$$

but

$$
\int d r\left|A_{V}(0, r)\right|\left|B_{V}(r, 0)\right| \leqq|A|\left|B^{+}\right|,
$$

hence, by Fubini

$$
M(A B)=\int d r \overline{A_{V}(0, r) B_{V}(r, 0)}=\int d r \overline{A_{V}(r, 0) B_{V}(0, r)}
$$

by translation invariance. Since $\int d r\left|A_{V}(r, 0)\right|\left|B_{V}(0, r)\right| \leqq\left|A^{+}\right||B|$, we can apply Fubini's theorem again, to conclude.

We can now define the conductivities, from the Kubo formula, in the infinite system, as given by

$$
\begin{aligned}
& \sigma_{x y}=\lim _{\varepsilon \downarrow 0} \frac{1}{i \varepsilon} \int_{0}^{+\infty} e^{-\varepsilon t} M\left(f\left[v_{x}, v_{y}(-t)\right] f\right) d t, \\
& \sigma_{y y}=\lim _{\varepsilon \downarrow 0} \frac{1}{i \varepsilon} \int_{0}^{+\infty} e^{-\varepsilon t} M\left(f\left[v_{y}, v_{y}(-t)\right] f\right)+\frac{1}{\varepsilon} M(f) .
\end{aligned}
$$

For fixed $\varepsilon$ at least, these formulas make sense. Indeed, from Proposition $6, f v_{x} \in \mathscr{C}$, and $f v_{y}(-t) \in \mathscr{C}$, so that by Proposition 2: $f\left[v_{x}, v_{y}(-t)\right] f \in \mathfrak{M}$. Similarly $f \in \mathfrak{M}$, since $f=f \cdot f$, and we can apply Proposition 2 .

We may also note that with probability one, the expression we give for the conductivity corresponds to the physical definition in terms of the space average of the local current induced by the adiabatic switching of the electric field. It might have been more natural however to define $\sigma_{x y}$, for example, by

$$
\sigma_{x y}=\lim _{\varepsilon \downarrow 0} \frac{1}{i \varepsilon} \int_{0}^{+\infty} e^{-\varepsilon t} M\left(f\left[v_{x}, v_{y}(-t)\right]\right) .
$$

But owing to the cyclicity property of $M$ and the fact that $f^{2}=f$ this is the same as the definition we give, to the extent that expression (2.21) makes sense. We have not proven this, in full generality however, and this is the reason why we take expressions (2.19) and (2.20) as the definitions of the conductivity. 


\section{The Gap Case}

We will first consider the case where the chemical potential $\mu$ lies in a gap. This is possible when $\|V\|<\frac{1}{2}$. Indeed, it follows immediately from the second resolvent equation and the explicit determination of the spectrum of $H_{0}(2.16)$, that $\sigma(H) \subset \bigcup_{n=0}^{\infty} A_{n}$. Let us define

$$
A=\int_{0}^{+\infty} e^{-\varepsilon t} v_{y}(-t) d t
$$

Using the spectral decomposition of $H$, we can write

$$
[A, f]=\int_{R^{2}} E(d \lambda) v_{y} E\left(d \lambda^{\prime}\right) \frac{f_{\lambda^{\prime}}-f_{\lambda}}{\varepsilon+i\left(\lambda-\lambda^{\prime}\right)},
$$

$E(d \lambda)$ being the spectral measure of $H$, and

$$
f_{\lambda}=\left\{\begin{array}{ll}
1 & \lambda<\mu \\
0 & \lambda \geqq \mu
\end{array} .\right.
$$

Taking for $\Gamma$, a contour (counterclockwise oriented) in the complex plane, crossing the real axis at $\pm \mu$, and encircling the points $\pm i \varepsilon$, we have the representation

$$
\frac{f_{\lambda^{\prime}}-f_{\lambda}}{\varepsilon+i\left(\lambda-\lambda^{\prime}\right)}=i \oint_{\Gamma} \frac{d z}{2 \pi i} \frac{1}{z-\lambda} \frac{1}{z-\lambda^{\prime}-i \varepsilon},
$$

so that we can rewrite

$$
[A, f]=i \oint_{\Gamma} \frac{d z}{2 \pi i} G_{z} v_{y} G_{z-i \varepsilon} .
$$

On the other hand

$$
\sigma_{x y}=\lim _{\varepsilon \downarrow 0} \frac{2 \operatorname{Im}}{\varepsilon} M\left(f v_{x} A f\right)=\lim _{\varepsilon \downarrow 0} \frac{2 \operatorname{Im}}{\varepsilon} M\left(f v_{x}[A, f] f\right),
$$

since $\operatorname{Im} M\left(f v_{x} f A f\right)=0$, because $A=A^{+}$. We can rewrite, from (3.5),

$$
[A, f]=i f_{y}-\varepsilon \oint_{\Gamma} \frac{d z}{2 \pi i} G_{z} v_{y} G_{z} G_{z-i \varepsilon},
$$

where

$$
f_{y}=\oint_{\Gamma} \frac{d z}{2 \pi i} G_{z} v_{y} G_{z} .
$$

Using the fact that

$$
v_{\substack{y \\ x}}=i\left[H-z, \frac{y}{x}\right]
$$

we see that

$$
f_{y}=i\left[f, \begin{array}{l}
y \\
x
\end{array}\right] .
$$


Hence

$$
f v_{x} f_{y}+f_{y} v_{x} f=i\left[f v_{x} f, y\right]
$$

From Propositions 5 and 1, we see from the expression (3.7) that $f_{y} \in \mathscr{C} \cap \mathfrak{B}$. Moreover, Propositions 1, 2, and 6 allow us to conclude that $f v_{x} f_{y} \in \mathfrak{M}, f_{y} v_{x} f \in \mathfrak{M}$, and $\left[f v_{x} f, y\right] \in \mathfrak{M}$. Using the cyclicity property of $M$, we can conclude that

$$
2 \operatorname{Re} M\left(f v_{x} f_{y} f\right)=2 \operatorname{Re} M\left(f v_{x} f_{y}\right)=0,
$$

and therefore that

$$
\sigma_{x y}=\lim _{\varepsilon \downarrow 0}-2 \operatorname{Im} M\left(f v_{x} \oint_{\Gamma} \frac{d z}{2 \pi i} G_{z} v_{y} G_{z} G_{z-i \varepsilon} f\right),
$$

which gives

$$
\sigma_{x y}=-2 \operatorname{Im} M\left(f v_{x} \oint_{\Gamma} \frac{d z}{2 \pi i} G_{z} v_{y} G_{z}^{2} f\right),
$$

since the remainder is bounded by:

$$
\varepsilon M^{1 / 2}\left(f v_{x}^{2} f\right) \frac{|\Gamma|}{2 \pi} \sup _{z \in \Gamma}|f|\left\|G_{z-i \varepsilon} G_{z}^{2} v_{y} G_{z}\right\|
$$

by Schwartz inequality and (2.10). It follows from Proposition 5 , that each term in (3.13) is bounded. Similarly

$$
\sigma_{y y}=\lim _{\varepsilon \downarrow 0} \frac{2 \operatorname{Re}}{\varepsilon} M\left(f v_{y} f_{y} f\right)+\frac{1}{\varepsilon} M(f)-2 \operatorname{Im} M\left(f v_{y} \oint_{\Gamma} \frac{d z}{2 \pi i} G_{z} v_{y} G_{z} G_{z-i \varepsilon} f\right) .
$$

Using the equation

$$
f v_{y} f_{y}+f_{y} v_{y} f=i\left[f v_{y} f, y\right]-f,
$$

derived from (3.9), we see that

$$
\sigma_{y y}=-2 \operatorname{Im} M\left(f v_{y} \oint_{\Gamma} \frac{d z}{2 \pi i} G_{z} v_{y} G_{z}^{2} f\right) .
$$

From Propositions 5 and 6 and the cyclicity of $M$, we can rewrite

$$
\sigma_{x y}=-2 \operatorname{Im} M\left(f v_{y} \oint_{\Gamma} \frac{d z}{2 \pi i} G_{z} v_{x} G_{z} v_{y} G_{z} f\right),
$$

and this can be expressed in the more transparent form, which will be the final one

$$
\sigma_{x y}=\frac{1}{i} M\left(f\left[f_{y}, f_{x}\right]\right)
$$

This can be seen as follows:

$$
f_{x} f_{y}=\oint_{\Gamma} \frac{d z}{2 \pi i} G_{z} v_{x} G_{z} \times \oint_{\Gamma^{\prime}} \frac{d z^{\prime}}{2 \pi i} G_{z^{\prime}} v_{y} G_{z^{\prime}}
$$


$\Gamma^{\prime}$ being a contour encircling $\Gamma$, but such that it still cuts the real axis in the resolvent set of $H$.

Using the second resolvent equation, we can rewrite this as

$$
f_{x} f_{y}=\oint_{\Gamma} \frac{d z}{2 \pi i} G_{z} v_{x} G_{z} v_{y} \oint_{\Gamma} \frac{d z^{\prime}}{2 \pi i} \frac{G_{z^{\prime}}}{z^{\prime}-z}-\oint_{\Gamma} \frac{d z}{2 \pi i} \frac{G_{z}}{z^{\prime}-z} \oint_{\Gamma} \frac{d z^{\prime}}{2 \pi i} v_{x} G_{z^{\prime}} v_{y} G_{z^{\prime}},
$$

but

$$
\begin{gathered}
\oint_{\Gamma^{\prime}} \frac{G_{z^{\prime}}}{z^{\prime}-z} \frac{d z^{\prime}}{2 \pi i}=G_{z}(1-f), \\
\oint_{\Gamma} \frac{G_{z}}{z^{\prime}-z} \frac{d z}{2 \pi i}=f G_{z^{\prime}},
\end{gathered}
$$

so that

$$
f_{x} f_{y} f=f \oint_{\Gamma^{\prime}} \frac{d z^{\prime}}{2 \pi i} G_{z^{\prime}} v_{x} G_{z^{\prime}} v_{y} G_{z^{\prime}} f
$$

This identity allows us to express $\sigma_{x y}$ both in the form (3.16) and (3.17). The same procedure gives

$$
\sigma_{y y}=0
$$

as expected.

In order to prove that $\sigma_{x y}$ as expressed in (3.17) is quantised, we would like to introduce an approximation scheme. Let $\Lambda$ be the square of side $L$ centered at the origin. Define as $V^{L}(r)$ a periodic potential equal to $V(r)$ in $\Lambda$, of period $L$, i.e. $V^{L}(x+L, y)=V^{L}(x, y+L)=V^{L}(x, y)$. Since $\left\|V^{L}\right\|=\|V\|<\frac{1}{2}$, when $\mu$ is in a gap of $H$, it is in a gap of $H^{L}$. For the system with the potential $V^{L}$, we can define the Hall conductivity as given by

$$
\sigma_{x y}^{L}=\frac{1}{i} M\left(f^{L}\left[f_{y}^{L}, f_{x}^{L}\right]\right)
$$

where

$$
\begin{gathered}
f^{L}=\oint_{\Gamma} \frac{d z}{2 \pi i} G_{z}^{L}, \\
f_{\substack{x \\
y}}^{L}=\oint_{\Gamma} \frac{d z}{2 \pi i} G_{z}^{L} v_{x} G_{z}^{L},
\end{gathered}
$$

and $M\left(A^{L}\right)$ means

$$
M\left(A^{L}\right)=\frac{1}{L^{2}} \int_{A} d^{2} r \overline{A_{V}^{L}(r, r)},
$$

which is well defined for all operators $A_{V}^{L}$ with a kernel continuous on $R^{2} \times R^{2}$, and such that $\overline{\left|A_{V}^{L}(r, r)\right|}<\infty$. We call $\mathfrak{M}^{L}$ the class of such operators. We will see that

$$
f^{L}\left[f_{y}^{L}, f_{x}^{L}\right] \in \mathfrak{M}^{L} .
$$


We can expect that

$$
\sigma_{x y}=\lim _{L \rightarrow \infty} \sigma_{x y}^{L},
$$

and we are going to prove this.

First of all, it is clear that if we drop the condition of translation invariance on the operators, we still have the analogue of Properties 1 and 2: $A^{L} \in \mathscr{C}, B^{L} \in \mathfrak{B}$, then $A^{L} B^{L} \in \mathscr{C}$, and $A^{L} \in \mathscr{C}, B^{L+} \in \mathscr{C}$, then $A^{L} B^{L} \in \mathfrak{M}^{L}$.

Moreover, if we call

$$
m\left(A^{L}\right)=M\left(A^{L} A^{+L}\right)^{1 / 2},
$$

then

$$
\left|M\left(A^{L} B^{L}\right)\right| \leqq m\left(A^{L}\right) m\left(B^{L+}\right),
$$

and

$$
m\left(A^{L} B^{L}\right) \leqq m\left(A^{L}\right) \sup _{\|V\| \text { fixed }}\left\|B_{V}^{L}\right\|,
$$

as a consequence of $(2.10)$.

We first prove the following technical:

Proposition 8. a) $f^{L}\left[f_{y}^{L}, f_{x}^{L}\right] \in \mathfrak{M}^{L}$.

b) The following quantity is finite

$$
a=\sup _{\substack{z \in \Gamma, L \\\|V\| \text { fixed }}}\left\{\left\|G_{z}^{L}\right\|,\left\|G_{z}^{L} v_{x}\right\|,\left\|f_{y}^{L}\right\|,\left\|f_{y}\right\|, m\left(f^{L}\right), m\left(G_{z}^{L}\right)\right\} .
$$

Proof. Since

$$
\left\|G_{z}^{L}\right\| \leqq \frac{1}{\operatorname{dist}\left(\mu, \sigma\left(H_{0}\right)+\|V\|\right)}
$$

and

$$
G_{z}^{L} v_{x}=\underset{y}{G_{z}^{0} v_{x}}+G_{z}^{L} V^{L} G_{z}^{0} v_{x}
$$

we see that

$$
\sup _{\substack{z \in \Gamma, L \\\|V\| \text { fixed }}}\left(\left\|G_{z}^{L}\right\|,\left\|G_{z}^{L} v_{x}\right\|,\left\|f_{y}^{L}\right\|\right)<\infty
$$

since $\sup _{z \in \Gamma}\left\|G_{z}^{0}\right\|<\infty$. The same properties hold for $G_{z}, G_{z} v_{x}, f_{x}$. On the other hand, since $\sup _{z \in \Gamma}\left|G_{z}^{0}\right|<\infty$ and $G_{z}^{L}=G_{z}^{0}+G_{z}^{0} V^{L} G_{z}^{L}$, we have

$$
\sup _{\substack{z \in \Gamma, L \\\|\boldsymbol{V}\| \text { fixed }}}\left|G_{z}^{L}\right|<\infty
$$

From these properties it follows that $f^{L}\left[f_{y}^{L}, f_{x}^{L}\right] \in \mathfrak{M}^{L}$, and

$$
\sup _{\substack{z \in \Gamma \\ L}} m\left(G_{z}^{L}\right) \leqq \sup _{\substack{z \in \Gamma, L \\\|V\| \text { fixed }}}\left|G_{z}^{L}\right|<\infty
$$


and finally

$$
m\left(f^{L}\right)<m\left(G_{z}^{L}\right)(1+\mu)<\infty .
$$

Consider now $\sigma_{x y}-\sigma_{x y}^{L}$. We can write

$$
\begin{aligned}
M\left(f f_{y} f_{x}\right)-M\left(f^{L} f_{y}^{L} f_{x}^{L}\right)= & M\left(\left(f-f^{L}\right) f_{y} f_{x}\right)+M\left(f^{L}\left(f_{y}-f_{y}^{L}\right) f_{x}\right) \\
& +M\left(f^{L} f_{y}^{L}\left(f_{x}-f_{x}^{L}\right)\right) .
\end{aligned}
$$

We will prove that each of these terms tends to zero, when $L$ tends to infinity. We will use repeatedly the following:

Proposition 9. If $A_{z} \in \mathscr{C}, \forall z \in \Gamma$ and $\sup _{z \in \Gamma} m\left(A_{z}\right)<\infty$, then

$$
\lim _{L \rightarrow \infty} \oint_{\Gamma} \frac{|d z|}{2 \pi} m\left(A_{z} \delta V^{L}\right)=0 \text {, }
$$

where $\delta V^{L}=V-V^{L}$.

Proof. Since $m\left(A_{z} \delta V^{L}\right) \leqq 2\|V\| \sup _{z \in \Gamma} m\left(A_{z}\right)$, it suffices, by dominated convergence to show that

$$
\lim _{L \rightarrow \infty} m\left(A_{z} \delta V^{L}\right)=0 \quad \forall z \in \Gamma
$$

But

$$
m^{2}\left(A_{z} \delta V^{L}\right)=\int_{\Lambda} \frac{d r}{L^{2}} \overline{\int d r^{\prime}\left|A_{z}\left(r, r^{\prime}\right)\right|^{2}} \delta V^{L}\left(r^{\prime}\right)^{2} \leqq \frac{4\|V\|^{2}}{L^{2}} \int_{\Lambda} d r \int_{R^{2} \backslash \Lambda} d r^{\prime} \overline{\left|A_{z}\left(0, r^{\prime}-r\right)\right|^{2}}
$$

and the right-hand side of this inequality tends to zero, by dominated convergence, since $\int d r \overline{\left|A_{z}(0, r)\right|^{2}}<\infty$.

Consider the first term of (3.32)

$$
M\left(\left(f-f^{L}\right) f_{y} f_{x}\right)=\oint_{\Gamma} \frac{d z}{2 \pi i} M\left(G_{z}^{L} \delta V^{L} G_{z} f_{y} f_{x}\right),
$$

we have

$$
\left|M\left(\left(f-f^{L}\right) f_{y} f_{x}\right)\right| \leqq a-\frac{|d z|}{\Gamma} m\left(f_{x} f_{y} G_{z^{*}} \delta V^{L}\right)
$$

and this tends to zero, from Proposition 9, since

$$
\sup _{z \in \Gamma} m\left(f_{x} f_{y} G_{z^{*}}\right) \leqq a^{2} m\left(f_{x}\right) .
$$

Similarly, the second term of (3.32) can be written as

$$
\begin{aligned}
& M(\left.f^{L}\left(f_{y}-f_{y}^{L}\right) f_{x}\right) \\
& \quad=\oint_{\Gamma} \frac{d z}{2 \pi i}\left[M\left(f^{L} G_{z}^{L} \delta V^{L} G_{z} v_{y} G_{z} f_{x}\right)+M\left(f^{L} G_{z}^{L} v_{y} G_{z}^{L} \delta V^{L} G_{z} f_{x}\right)\right] .
\end{aligned}
$$


Therefore

$$
\left.\mid M\left(f^{L} f_{y}-f_{y}^{L}\right) f_{x}\right) \mid \leqq a^{3} \oint \frac{|d z|}{2 \pi}\left[m\left(f_{x} G_{z^{*}} v_{y} G_{z^{*}} \delta V^{L}\right)+m\left(f_{x} G_{z^{*}} \delta V^{L}\right)\right]
$$

and this tends to zero, from Proposition 9, since each term satisfies the condition.

Finally, the last term of (3.32) can be written as

$$
\begin{aligned}
M\left(f^{L} f_{y}{ }_{y}\left(f_{x}-f_{x}^{L}\right)\right)= & \oint_{\Gamma} \frac{d z}{2 \pi i}\left[M\left(f^{L} f_{y}^{L} G_{z}^{L} \delta V^{L} G_{z} v_{x} G_{z}\right)\right. \\
& \left.+M\left(f^{L} f_{y}^{L} G_{z}^{L} v_{x} G_{z}^{L} \delta V^{L} G_{z}\right)\right]
\end{aligned}
$$

therefore

$$
\left|M\left(f^{L} f_{y}^{L}\left(f_{x}-f_{x}^{L}\right)\right)\right| \leqq a^{3} \oint_{\Gamma} \frac{|d z|}{2 \pi}\left[m\left(G_{z^{*}} v_{x} G_{z^{*}} \delta V^{L}\right)+m\left(G_{z^{*}} \delta V^{L}\right) a\right],
$$

which tends also to zero, from Proposition 9. Treating in the same way

$$
M\left(f f_{x} f_{y}\right)-M\left(f^{L} f_{x}^{L} f_{y}^{L}\right),
$$

we conclude that

$$
\sigma_{x y}=\lim _{L \rightarrow \infty} \sigma_{x y}^{L} .
$$

Finally note that we also have:

$$
M(f)=\lim _{L \rightarrow \infty} M\left(f^{L}\right)
$$

We would like to express now the periodic Hall conductivity, in a more transparent way. Choosing $L$, in such a way that $\frac{L^{2}}{2 \pi}$ is an integer, the magnetic translation operators $T(\underline{n} L)$ form an abelian group, which commutes with $H^{L}$. We can therefore apply Bloch theorem. $H^{L}$ is unitarily equivalent to

$$
\frac{1}{K^{2}} \int_{0}^{K} d k_{x} \int_{0}^{K} d k_{y} H^{L}(k)
$$

where $H^{L}(k)$ is the operator

$$
H^{L}(k)=\frac{1}{2}\left(\frac{1}{i} \partial_{x}+y+k_{x}\right)^{2}+\frac{1}{2}\left(\frac{1}{i} \partial_{y}+k_{y}\right)^{2}+V^{L}(x, y)
$$

defined on $L^{2}(\Lambda)$, with the boundary conditions

$$
\varphi(L, y)=\varphi(0, y), \quad \varphi(x, L)=e^{-i L x} \varphi(x, 0),
$$

and

$$
\begin{aligned}
\varphi_{x}(L, y) & =\varphi_{x}(0, y), \quad \varphi_{y}(L, y)=\varphi_{y}(0, y), \\
\varphi_{x}(x, L)+i L \varphi(x, L) & =e^{-i L x} \varphi_{x}(x, 0), \quad \varphi_{y}(x, L)=e^{-i L x} \varphi_{y}(x, 0),
\end{aligned}
$$

and

$$
K=\frac{2 \pi}{L}
$$


One can see that $G_{z}^{L}(k)=\left(z-H^{L}(k)\right)^{-1}$ is compact for all $k$. Since $f\left[f_{y}, f_{x}\right]$ commute with $T(\underline{n} L)$, we can write the Hall conductivity in the periodic system as

$$
\sigma_{x y}^{L}=\frac{1}{i K^{2} L^{2}} \int_{0}^{K} d k_{x} \int_{0}^{K} d k_{y} \overline{\operatorname{Tr} f^{L}(k)\left[f_{y}^{L}(k), f_{x}^{L}(k)\right]}
$$

where $f^{L}(k)$ is the projector

and

$$
f^{L}(k)=\oint_{\Gamma} \frac{d z}{2 \pi i} G_{z}^{L}(k)
$$

$$
f_{x}^{L}(k)=\oint_{\Gamma} \frac{d z}{2 \pi i} G_{z}^{L}(k)\left(\underset{y}{x}+\underset{y}{k_{x}}\right) G_{z}^{L}(k) .
$$

Equation (3.38) is valid, because $f^{L}(k)\left[f_{y}^{L}(k), f_{x}^{L}(k)\right] \in \mathfrak{M}^{L}$ and $f^{L}(k)$ is trace class. Since

$$
\underset{x}{v_{x}}+\underset{y}{k_{x}}=\underset{y}{\partial k_{x}} H^{L}(k)
$$

and $G_{z}^{L}(k)$ is $c^{\prime}$ in $k$, we can write (3.40) more simply as

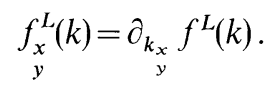

Introducing the operator

$$
\hat{H}^{L}(k)=\frac{1}{2}\left(\frac{1}{i} \partial_{x}+y\right)^{2}+\frac{1}{2}\left(\frac{1}{i} \partial_{y}\right)^{2}+V^{L}(x, y)
$$

defined on $L^{2}(\Lambda)$, with the boundary conditions

$$
\varphi(L, y)=e^{i k_{x} L} \varphi(0, y), \quad \varphi(x, L)=e^{i k_{y} L-i L x} \varphi(x, 0),
$$

and

$$
\begin{gathered}
\varphi_{x}(L, y)=e^{i k_{x} L} \varphi_{x}(0, y), \quad \varphi_{y}(L, y)=e^{i k_{y} L} \varphi_{y}(0, y), \\
\varphi_{x}(x, L)+i L \varphi(x, L)=e^{i k_{y} L-i L x} \varphi_{x}(x, 0), \quad \varphi_{y}(x, L)=e^{i k_{y} L-i L x} \varphi_{y}(x, 0),
\end{gathered}
$$

we see that the projector

$$
P^{L}(k)=\oint_{\Gamma} \frac{d z}{2 \pi i}\left(z-\hat{H}^{L}(k)\right)^{-1}
$$

is related to the old one $f^{L}(k)$, by

$$
P^{L}(k)=e^{i k \cdot r} f^{L}(k) e^{-i k \cdot r} .
$$

But $P^{L}(k)$ is periodic in $k$ :

$$
P^{L}\left(k_{x}+K, k_{y}\right)=P^{L}\left(k_{x}, k_{y}+K\right)=P^{L}\left(k_{x}, k_{y}\right) .
$$

Using (3.46), a simple computation shows that

$$
\begin{aligned}
\operatorname{Tr} f^{L}\left[\partial_{k_{y}} f^{L}, \partial_{k_{x}} f^{L}\right]= & \operatorname{Tr} P^{L}\left[\partial_{k_{y}} P^{L}, \partial_{k_{x}} P^{L}\right] \\
& +i\left(\partial_{k_{x}} \operatorname{Tr} P^{L} y-\partial_{k_{y}} \operatorname{Tr} P^{L} x\right) .
\end{aligned}
$$


Therefore, since $P^{L}(k)$ is periodic in $k$, we can rewrite the Hall conductivity as:

$$
\sigma_{x y}^{L}=\frac{1}{i(2 \pi)^{2}} \int_{0}^{K} d k_{x} \int_{0}^{K} d k_{y} \operatorname{Tr} P^{L}(k)\left[\partial_{k_{y}} P^{L}, \partial_{k_{x}} P^{L}\right] .
$$

Once we have put the conductivity in this form, it is possible to show that it is quantised, i.e.

$$
\sigma_{x y}^{L}=\frac{1}{2 \pi} \times \text { integer }
$$

the integer being a topological invariant. This is possible by recognising in it the first Chern number of a complex vector bundle [induced by $P^{L}(k)$, on the 2-torus]. But it is also possible to give a direct and simple proof of this quantisation, and this is what we do in the next paragraph.

Note that since $\sigma_{x y}^{L}$ converges to $\sigma_{x y},(3.50)$ tells us that $\sigma_{x y}=\frac{1}{2 \pi} \times$ integer. The remaining task is to compute this integer. The expression (3.17) for $\sigma_{x v}$ shows that this quantity is continuous in $\lambda$, if we replace the potential $V$ by $\lambda V$, as long as $\lambda \in[0,1]$. Taking $\lambda=0$, we are back to the free case, for computing this integer. When there is no potential, the equations of motion give

$$
v_{x}(t)=v_{x} \cos t+v_{y} \sin t, \quad v_{y}(t)=-v_{x} \sin t+v_{y} \cos t .
$$

Putting back this result, in the expression (2.19) for $\sigma_{x y}$, and using the fact that $\left[v_{x}, v_{y}\right]=i(3.52)$, one finds

$$
\sigma_{x y}=M\left(f_{0}\right)=\frac{1}{2 \pi} \sum_{n: n+\frac{1}{2}<\mu} .
$$

On the other hand, the electronic density $\varrho$ is given in a gap by:

$$
\varrho=M(f)=\frac{1}{2 \pi i} \oint_{\Gamma} d z M\left(G_{z}\right) .
$$

Replacing the potential $V$ by $\lambda V$, we get from (3.53),

$$
\frac{d \varrho}{d \lambda}=\frac{1}{2 \pi i} \oint_{\Gamma} d z M\left(G_{z} V G_{z}\right)=\frac{1}{2 \pi i} M\left(\oint_{\Gamma} d z G_{z}^{2} V\right)=0
$$

from the cyclicity property of $M$. Therefore

$$
\varrho=M\left(f_{0}\right)=\frac{1}{2 \pi} \sum_{n: n+\frac{1}{2}<\mu} .
$$

In other words, we have proven that

$$
\sigma_{x y}=\frac{1}{2 \pi} N,
$$

when

$$
\varrho=\frac{1}{2 \pi} N,
$$

$N$ being the Landau band index. 


\section{The Topological Invariant}

We give here a simple proof of the quantisation. We consider now the $m$-dimensional projectors $P^{L}(k)$, as operators defined for all values of $k \in \mathbb{R}^{2}$ such that

$$
P^{L}\left(k+K_{\alpha}\right)=P^{L}(k)
$$

where

$$
K_{1}=(K, 0), \quad K_{2}=(0, K) .
$$

Since $P^{L}(k)$ is $C^{2}$ in $k$, a theorem of Kato [24] ensures us that there exist $m$ functions $\left\{\psi_{j}(k)\right\}_{j=1}^{m}, c^{2}$ in $k$, forming an orthonormal basis of the subspace projected on by $P^{L}(k)$. The periodicity property of $P^{L}(k)$, induces the following transformation of this basis:

$$
\psi_{j}\left(k+K_{\alpha}\right)=\sum_{e} \psi_{e}(k) S_{e j}^{\alpha}(k)
$$

$S^{\alpha}(k)$ being a unitary $m \times m$ matrix given by

$$
S_{e j}^{\alpha}(k)=\left(\psi_{e}(k), \psi_{j}\left(k+K_{\alpha}\right)\right) .
$$

Let us now introduce the following "gauge field" $m \times m$ matrix: $A_{\beta}(k)$,

$$
A_{\beta}^{i j}(k)=\left(\psi_{i}(k), \partial_{k_{\beta}} \psi_{j}(k)\right) \text {. }
$$

In a translation of the $k$ 's by $K_{\alpha}$, they transform as:

$$
A_{\beta}\left(k+K_{\alpha}\right)=\left(S^{\alpha}\right)^{+} \partial_{k_{\beta}} S^{\alpha}+\left(S^{\alpha}\right)^{+} A_{\beta}(k) S^{\alpha}
$$

as a consequence of the transformation law (4.3), (4.4), of the $\psi_{j}(k)$.

The usefulness of these matrices comes from the fact that we can write

$$
\operatorname{Tr} P^{L}\left[\partial_{k_{1}} P^{L}, \partial_{k_{2}} P^{L}\right]=\partial_{k_{1}} \operatorname{tr} A_{2}-\partial_{k_{2}} \operatorname{tr} A_{1},
$$

therefore the transformation law (4.6) for the $A$ 's implies that if

$$
I=\int_{0}^{K} d k_{1} \int_{0}^{K} d k_{2} \operatorname{Tr} P^{L}\left[\partial_{k_{1}} P^{L}, \partial_{k_{2}} P^{L}\right]
$$

then

$$
I=\int_{0}^{K} d k_{2}\left\{\operatorname{tr}\left(S^{1}\right)^{+} \partial_{k_{2}} S^{1}\right\}\left(0, k_{2}\right)-\int_{0}^{K} d k_{1}\left\{\operatorname{tr}\left(S^{2}\right)^{+} \partial_{k_{1}} S^{2}\right\}\left(k_{1}, 0\right)
$$

Defining $\phi^{\alpha}(k)$ by $S^{\alpha}(k)=\exp i \phi^{\alpha}(k)$, the cyclicity of the trace implies that

$$
I=i \operatorname{tr} \phi^{1}\left(K_{2}\right)-\phi^{1}(0)-\phi^{2}\left(K_{1}\right)+\phi^{2}(0) \text {. }
$$

On the other hand, expressing $\psi_{j}\left(k+K_{1}+K_{2}\right)$ either as the result of a translation by $K_{1}$, followed by one by $K_{2}$, or the converse, we get the following compatibility condition for the matrices $S$ :

$$
\left(S^{\alpha}\right)^{+}(k) S^{\beta}(k) S^{\alpha}\left(k+K_{\beta}\right)\left(S^{\beta}\left(k+K_{\alpha}\right)\right)^{+}=\mathbb{1} .
$$


Equating the determinant of both sides of this equation and using the fact that $\operatorname{det} S^{\alpha}(k)=\exp i \operatorname{tr} \phi^{\alpha}(k)$, we see that

$$
\operatorname{tr}-\phi^{\alpha}(k)+\phi^{\beta}(k)+\phi^{\alpha}\left(k+K_{\beta}\right)-\phi^{\beta}\left(k+K_{\alpha}\right)=2 \pi \times \text { integer, }
$$

and this leads to the desired result,

$$
I=2 \pi i \times \text { integer . }
$$

\section{The Localised States Case}

We would like to consider now the case where the chemical potential $\mu$ lies in a region of localised states. To fix ideas let us take

$$
\mu \in A_{n}^{+}=\left[n+\frac{1}{2}+\|V\|-\hat{\partial}_{n}, n+\frac{1}{2}+\|V\|\right] .
$$

We will first decompose the conductivity $\sigma_{x y}$ into two contributions:

$$
\sigma_{x y}=\sigma_{x y}^{\mathrm{I}}+\sigma_{x y}^{\mathrm{II}},
$$

where $\sigma_{x y}^{\mathrm{I}}$ would be the conductivity if $\mu$ was in a gap (of index $n$ ) and $\sigma_{x y}^{\mathrm{II}}$ is a contribution coming essentially from localised states. The same decomposition holds for $\sigma_{y y}$.

Let $\mu_{n}$ be any point in the gap of index $n$, i.e.

$$
\mu_{n} \in\left(n+\frac{1}{2}+\|V\|, n+\frac{3}{2}-\|V\|\right) .
$$

Then we can write:

$$
f=f_{n}-\chi_{n},
$$

where $f_{n}=E\left(-\infty, \mu_{n}\right)$,

$$
\chi_{n}=E\left(\mu, \mu_{n}\right)=E\left(\mu, n+\frac{1}{2}+\|V\|\right) .
$$

Now, it follows from the fact that $f_{n} \chi_{n}=\chi_{n}$ and the cyclicity property of $M$, that if $A$ is any translation invariant observable, that:

$$
M(f A f)=M\left(f_{n} A f_{n}\right)-M\left(\chi_{n} A \chi_{n}\right) .
$$

This proves the decomposition (5.2) for the conductivities. $\sigma_{x y}^{\mathrm{I}}$ and $\sigma_{y y}^{\mathrm{I}}$ being given by the expressions we have discussed previously ( $\mu_{n}$ being in a gap) and the remaining ones being given by

$$
\begin{aligned}
& \sigma_{x y}^{\mathrm{II}}=\lim _{\varepsilon \downarrow 0} \frac{-2 \operatorname{Im}}{\varepsilon} \int_{0}^{+\infty} e^{-\varepsilon t} M\left(\chi_{n} v_{x} v_{y}(-t) \chi_{n}\right), \\
& \sigma_{y y}^{\mathrm{II}}=\lim _{\varepsilon \downarrow 0} \frac{-2 \operatorname{Im}}{\varepsilon} \int_{0}^{+\infty} e^{-\varepsilon t} M\left(\chi_{n} v_{y} v_{y}(-t) \chi_{n}\right)-\frac{1}{\varepsilon} M\left(\chi_{n}\right) .
\end{aligned}
$$

Since we assume that the spectrum of $H$ in the interval $B=\left[\mu, n+\frac{1}{2}+\|V\|\right]$ is pure point only with probability one, we have

$$
\chi_{n}=\sum_{\lambda \in B} E_{\lambda},
$$


$E_{\lambda}$ being the spectral projector of $H$ corresponding to the eigenvalue $\lambda$. It is easily seen, that if $A \in \mathscr{C}$, then

$$
M\left(\sum_{\lambda \in B} E_{\lambda} A E_{\lambda}\right)=M\left(A \sum_{\lambda \in B} E_{\lambda}\right) .
$$

Therefore, using Proposition 6, we can write

$$
M\left(\chi_{n} v_{x} v_{y}(-t) \chi_{n}\right)=M\left(\sum_{\lambda \in B} E_{\lambda} v_{x} v_{y}(-t) E_{\lambda}\right)
$$

On the other hand, using the relationships

$$
v_{x}=i\left[H-\lambda, \begin{array}{l}
x \\
y
\end{array}\right]
$$

we can write

$$
\begin{aligned}
E_{\lambda} \int_{0}^{+\infty} e^{-\varepsilon t} v_{x} v_{y}(-t) d t E_{\lambda}= & -E_{\lambda} x v_{y} E_{\lambda}+\varepsilon E_{\lambda} x y E_{\lambda} \\
& -\varepsilon^{2} E_{\lambda} x \frac{1}{\varepsilon+i(H-\lambda)} y E_{\lambda}
\end{aligned}
$$

To the extent that all terms are finite, we have therefore

$$
\begin{aligned}
\sigma_{x y}^{\mathrm{II}}= & \lim _{\varepsilon \downarrow 0}\left\{\frac{2 \operatorname{Im}}{\varepsilon} M\left(\sum_{\lambda \in B} E_{\lambda} x v_{y} E_{\lambda}\right)-2 \operatorname{Im} M\left(\sum_{\lambda \in B} E_{\lambda} x y E_{\lambda}\right)\right\} \\
& +\lim _{\varepsilon \downarrow 0} 2 \varepsilon \operatorname{Im} M\left(\sum_{\lambda \in B} E_{\lambda} x \frac{1}{\varepsilon+i(H-\lambda)} y E_{\lambda}\right),
\end{aligned}
$$

and

$$
\begin{aligned}
\sigma_{y y}^{\mathrm{II}}= & \lim _{\varepsilon \downarrow 0} \frac{1}{\varepsilon}\left\{2 \operatorname{Im} M\left(\sum_{\lambda \in B} E_{\lambda} y v_{y} E_{\lambda}\right)-M\left(\sum_{\lambda \in B} E_{\lambda}\right)\right\} \\
& +\lim _{\varepsilon \downarrow 0} 2 \varepsilon \operatorname{Im} M\left(\sum_{\lambda \in B} E_{\lambda} y \frac{1}{\varepsilon+i(H-\lambda)} y E_{\lambda}\right),
\end{aligned}
$$

and since $\left[x, v_{y}\right]=[x, y]=0$ and $\left[y, v_{y}\right]=i$, we can rewrite these expressions in the form

$$
\sigma_{y y}^{\mathrm{II}}=\lim _{\varepsilon \downarrow 0} 2 \varepsilon \operatorname{Im} M\left(\sum_{\lambda \in B} E_{\lambda}^{x} \frac{x}{y+i(H-\lambda)} y E_{\lambda}\right) .
$$

It remains, however, to check the finiteness of all the expressions appearing in $\sigma_{x y}^{\text {II }}$ (5.14) and $\sigma_{y y}^{\mathrm{II}}(5.15)$, for fixed $\varepsilon$.

This can be seen as a consequence of the following inequalities:

$$
\left|M\left(\sum_{\lambda \in B} E_{\lambda} A C E_{\lambda}\right)\right| \leqq M^{1 / 2}\left(\sum_{\lambda \in B} E_{\lambda} A A^{+} E_{\lambda}\right) M^{1 / 2}\left(\sum_{\lambda \in B} E_{\lambda} C^{+} C E_{\lambda}\right),
$$

which is a version of Schwartz inequality and

$$
0 \leqq M\left(\sum_{\lambda \in B} E_{\lambda} A E_{\lambda}\right) \leqq M\left(\sum_{\lambda \in B} E_{\lambda} C E_{\lambda}\right)
$$


which holds if

$$
0 \leqq A=A^{+} \leqq C=C^{+} .
$$

By assumption, the localised states in $B$ have a finite localisation length, so that

$$
K(B)=\int d^{2} r r^{2} \overline{\sum_{\lambda \in B}\left|E_{\lambda}(0, r)\right|^{2}}=M\left(\sum_{\lambda \in B} E_{\lambda} r^{2} E_{\lambda}\right)<\infty .
$$

Hence

$$
\left|M\left(\sum_{\lambda \in B} E_{\lambda} x v_{y} E_{\lambda}\right)\right| \leqq M^{1 / 2}\left(\sum_{\lambda \in B} E_{\lambda} r^{2} E_{\lambda}\right) M^{1 / 2}\left(\sum_{\lambda \in B} E_{\lambda} \chi_{n} v_{y}^{2} \chi_{n} E_{\lambda}\right)<\infty
$$

by (5.20) and the fact that $\chi_{n} v_{y}^{2} \chi_{n} \in \mathscr{C}$, by Propositions 1 and 6 . Similarly

$$
\left|M\left(\sum_{\lambda \in B} E_{\lambda} x y E_{\lambda}\right)\right| \leqq K(B)<\infty,
$$

and

$$
\begin{aligned}
& \left|M\left(\sum_{\lambda \in B} E_{\lambda} x \frac{\varepsilon}{\varepsilon+i(H-\lambda)} y E_{\lambda}\right)\right| \\
& \quad \leqq M^{1 / 2}\left(\sum_{\lambda \in B} E_{\lambda} r^{2} E_{\lambda}\right) M^{1 / 2}\left(\sum_{\lambda \in B} E_{\lambda} y \frac{\varepsilon^{2}}{\varepsilon^{2}+(H-\lambda)^{2}} y E_{\lambda}\right)<\infty,
\end{aligned}
$$

since

$$
M\left(\sum_{\lambda \in B} E_{\lambda} y \frac{\varepsilon^{2}}{\varepsilon^{2}+(H-\lambda)^{2}} y E_{\lambda}\right) \leqq M\left(\sum_{\lambda \in B} E_{\lambda} y^{2} E_{\lambda}\right) \leqq K(B)
$$

by (5.18). The corresponding terms in $\sigma_{y y}^{\mathrm{II}}$ are treated in the same way.

On the other hand, there exist a $\delta>0$, such that the domain $|\lambda-H| \leqq \delta$ (in the operator sense) corresponds to a neighborhood $B_{\delta}$ of $B$, such that the spectrum of $H$ is pure point in $B_{\delta}$ and the localisation length is finite. We can therefore further decompose the conductivities as follows:

$$
\underset{\substack{x y \\ y y}}{\mathrm{II}}=\underset{x y}{\sigma_{x y}^{\mathrm{II}, 1}}+\underset{\substack{x y \\ y y}}{\mathrm{II}^{\mathrm{II}, 2}}+\underset{\substack{x y \\ y y}}{\mathrm{II}, 3}
$$

where

$$
\begin{gathered}
\sigma_{\substack{x y \\
y y}}^{\mathrm{II}, 1}=2 \operatorname{Im} M\left(\sum_{\lambda \in B} E_{\lambda} E_{y}^{x} E_{\lambda} y E_{\lambda}\right), \\
\sigma_{\substack{x y \\
y y}}^{\mathrm{II}, 2}=\lim _{\varepsilon \downarrow 0} 2 \operatorname{Im} M\left(\sum_{\substack{\lambda \in B \\
\lambda^{\prime} \in B_{\delta} \\
\lambda^{\prime} \neq \lambda}} E_{\lambda}^{x} E_{\lambda^{\prime}} y E_{\lambda} \frac{\varepsilon}{\varepsilon+i\left(\lambda^{\prime}-\lambda\right)}\right), \\
\sigma_{\substack{x y \\
y y}}^{\mathrm{II}, 3}=\lim _{\varepsilon \downarrow 0} 2 \operatorname{Im} M\left(\sum_{\lambda \in B} E_{\lambda}^{x}{ }_{y}^{x} Q_{\lambda} y E_{\lambda}\right)
\end{gathered}
$$


where

$$
\begin{array}{ll}
Q_{\lambda}=\frac{\varepsilon}{\varepsilon+i(H-\lambda)} & \text { if }|\lambda-H| \geqq \delta, \\
Q_{\lambda}=0 & \text { otherwise. }
\end{array}
$$

We now prove that $\sigma_{\substack{x y \\ y y}}^{\mathrm{II}, 2}$ and $\sigma_{\substack{x y \\ y y}}^{\mathrm{II}, 3}$ vanish.

From (5.17) and (5.18) it follows that

$$
\left|\begin{array}{c}
\sigma_{x y}^{\mathrm{II}, 3} \\
y y
\end{array}\right| \leqq \lim _{\varepsilon \downarrow 0} 2 K^{1 / 2}(B) M^{1 / 2}\left(\sum_{\lambda \in B} E_{\lambda} y Q_{\lambda}^{+} Q_{\lambda} y E_{\lambda}\right)
$$

but

$$
M\left(\sum_{\lambda \in B} E_{\lambda} y Q_{\lambda}^{+} Q_{\lambda} y E_{\lambda}\right) \leqq \frac{\varepsilon^{2}}{\delta^{2}} K(B)
$$

by (5.18) and (5.20).

On the other hand, Schwartz inequality gives

$$
\left|\sigma_{x y}^{\mathrm{II}, 2}\right| \leqq \lim _{\varepsilon \downarrow 0} 2 M^{1 / 2}\left(\sum_{\substack{\lambda \in B \\ \lambda^{\prime} \in B_{j} \\ \lambda^{\prime} \neq \lambda}} E_{\lambda} x E_{\lambda^{\prime}} x E_{\lambda} \frac{\varepsilon^{2}}{\varepsilon^{2}+\left(\lambda^{\prime}-\lambda\right)^{2}}\right) M^{1 / 2}\left(\sum_{\substack{\lambda \in B \\ \lambda^{\prime} \in B_{\delta} \\ \lambda^{\prime} \neq \lambda}} E_{\lambda} y E_{\lambda^{\prime}} y E_{\lambda}\right)
$$

But by (5.18)

$$
M\left(\sum_{\substack{\lambda \in B \\ \lambda^{\prime} \in B_{\delta} \\ \lambda^{\prime} \neq \lambda}} E_{\lambda} y E_{\lambda^{\prime}} y E_{\lambda}\right) \leqq M\left(\sum_{\lambda \in B} E_{\lambda} y\left(1-E_{\lambda}\right) y E_{\lambda}\right)
$$

and

$$
\begin{aligned}
M\left(\sum_{\lambda \in B} E_{\lambda} y\left(1-E_{\lambda}\right) y E_{\lambda}\right) & =\int d r^{\prime} d r^{\prime \prime} \overline{\sum_{\lambda \in B} E_{\lambda}\left(0, r^{\prime}\right) E_{\lambda}\left(r^{\prime}, r^{\prime \prime}\right) E_{\lambda}\left(r^{\prime \prime}, 0\right)}\left(\frac{y^{\prime}-y^{\prime \prime}}{2}\right)^{2} \\
& =\frac{1}{2} \int d r \overline{\sum_{\lambda \in B}\left|E_{\lambda}(0, r)\right|^{2} y^{2}}=\frac{K(B)}{2}<\infty .
\end{aligned}
$$

We can therefore conclude from the dominated convergence theorem that

$$
\sigma_{\substack{x y \\ y y}}^{\mathrm{II}, 2}=0
$$

We have thus proven that

$$
\sigma_{x y}^{\mathrm{II}}=2 \operatorname{Im} M\left(\sum_{\lambda \in B} E_{\lambda} x E_{\lambda} y E_{\lambda}\right)
$$

and

$$
\sigma_{y y}^{\mathrm{II}}=2 \operatorname{Im} M\left(\sum_{\lambda \in B} E_{\lambda} y E_{\lambda} y E_{\lambda}\right) .
$$

Equation (5.27) gives the expected result $\sigma_{y y}^{\mathrm{II}}=0$. Sufficiently localised states do not contribute to the parallel conductivity. But (5.26), without further assumption does 
not lead to $\sigma_{x y}^{\mathrm{II}}=0$. For a time reversal hamiltonian, the eigenfunctions can be taken real and in this case $\sigma_{x y}^{\mathrm{II}}=0$. But when a magnetic field is present, the eigenfunctions will be complex and $\sigma_{x y}^{\mathrm{II}}$ can be non-vanishing in general. That it can be so, can be seen by considering the free case: $E_{n+\frac{1}{2}}$ is of infinite dimension, and $K(B)<\infty$, for any $B$ containing only $n+\frac{1}{2}$, and in this case it can be checked explicitly that $\sigma_{x y}^{\mathrm{II}}=\frac{n+1}{2 \pi}$, if we take (5.26) for it.

A simple condition which guarantees that the contribution to the Hall conductivity coming from localised states is vanishing, is to assume that the eigenvalues are simple with probability one, besides the finiteness of the localisation length. In this case, we have indeed

$$
\sigma_{x y}^{\mathrm{II}}=2 \operatorname{Im} M\left(\sum_{\lambda \in B}\left|\varphi_{\lambda}(0)\right|^{2}\right)\left(\int\left|\varphi_{\lambda}^{\prime}(r)\right|^{2} x d r\right)\left(\int d^{2} r^{\prime}\left|\varphi_{\lambda}^{2}\left(r^{\prime}\right)\right| y^{\prime}\right)=0 .
$$

We think that this careful discussion of the Hall conductivity in the case of localised states shows the essential difference between this quantity and the more familiar parallel conductivity. In any case, the assumption of a non-degenerate point spectrum is a very natural one and can be expected to be always satisfied, as could be proven to be the case in all the situations where localised states were proven to exist.

We have thus proven that when the chemical potential $\mu$ lies in a gap or in a region of localised states, the Hall conductivity is quantised whereas the parallel one vanishes. Finally, if we describe the results in terms of the electronic density $\varrho(\mu)=M(f)$, we will have the announced result.

If all states in $A_{n}^{+}$and $A_{n+1}^{-}$are localised in the sense of $H_{1}$ and $H_{2}$, then if

$$
\begin{gathered}
\varrho_{-, n}=\varrho\left(n+\frac{1}{2}+\|V\|-\delta_{n}\right), \\
\varrho_{+, n}=\varrho\left(n+\frac{3}{2}-\|V\|+\delta_{n+1}\right) \\
\varrho_{-, n} \neq \varrho_{+, n} \quad \text { and } \quad \frac{n+1}{2 \pi}=\varrho\left(n+\frac{1}{2}+\frac{3}{4}\|V\|\right) \in\left(\varrho_{-, n}, \varrho_{+, n}\right) .
\end{gathered}
$$

Moreover, when $\varrho \in\left(\varrho_{-, n}, \varrho_{+, n}\right)$, then

$$
\sigma_{x y}=\frac{1}{2 \pi}(n+1), \quad \sigma_{y y}=0 .
$$

Finally, let us assume that all states are localised in each Landau band, with a finite localisation length, i.e. $K\left(A_{n}\right)<\infty$. Then our proof shows that we would have $\sigma_{x y}\left(\mu_{n+1}\right)=\sigma_{x y}\left(\mu_{n}\right)$, where $\mu_{n}$ is any point in the gap of index $n$, and $\mu_{n+1}$ a point in the gap of index $n+1$. This contradicts the results established previously $\sigma_{x y}\left(\mu_{n+1}\right)$ $-\sigma_{x y}\left(\mu_{n}\right)=\frac{1}{2 \pi}$. Consequently $K\left(A_{n}\right)=\infty$ for all $n \geqq 0$.

\section{Conclusion}

Our proof of the quantisation relies on the condition $\|V\|<\frac{1}{2}$. In a certain sense this condition is natural, since this means either weak disorder and strong 
magnetic field, and at strong disorder we could expect complete localisation with a finite localisation length and therefore a vanishing Hall conductivity. On the other hand, we could expect that we always have $\sigma_{x y}=\frac{1}{2 \pi} \times$ integer when $\mu$ is in the region of localised states, with a finite localisation length. A first step in the understanding of this situation would be to consider our model when $\|V\|>\frac{1}{2}$. There are no more gaps, but if $\mu$ is in the point spectrum, with probability one, it is not an eigenvalue. Therefore, if we approximate the hamiltonian, as we did, by a periodic one with a sufficiently large period $L$, then with probability one, $\mu$ will be in a gap of the periodic hamiltonian. But then the Hall conductivity of the periodic hamiltonian is of the form $\sigma_{x y}^{L}=\frac{1}{2 \pi} n^{L}, n^{L}$ being an integer. If one could show that $\sigma_{x y}^{L}$ converges to $\sigma_{x y}$, when $L$ tends to infinity, we would get the desired quantisation. Finally, we might expect again that when $\mu$ is in point spectrum, $\sigma_{x y}$ is continuous in the potential (as long as changing the potential doesn't change the nature of the spectrum). If it is so, we would prove that the integer is the same as the one we have computed. All these arguments make the conjecture very plausible, but we failed to turn them into a rigorous proof.

Acknowledgements. I would like to thank Ch. Ed. Pfister and G. Zumbach for many helpful discussions. This work is supported by the Fonds National Suisse de la Recherche Scientifique.

\section{References}

1. Von Klitzing, K., Dorda, G., Pepper, M.: New method for high-accuracy determination of the fine-structure of constant based on quantized Hall resistance. Phys. Rev. Lett. 45, 494 (1980)

2. Laughlin, R.B.: Quantized Hall conductivity in two dimensions. Phys. Rev. B 23, 5632 (1981)

3. Halperin, B.I.: Quantized Hall conductance current-carrying edge states, and the existence of extended states in a two-dimensional disordered potential. Phys. Rev. B 25, 2185 (1982)

4. Thouless, D.J., Kohmoto, M., Nightingale, P.P., Deh-Nijs, M.: Quantized Hall conductance in a two-dimensional periodic potential. Phys. Rev. Lett. 49, 1405 (1982)

5. Avron, J.E., Seiler, R., Simon, B.: Homotopy and quantization in condensed matter physics. Phys. Rev. Lett. 51, 51 (1983)

6. Dubrovin, B.A., Novikov, S.P.: Ground states of a two-dimensional electron in a periodic magnetic field. Sov. Phys. JETP 52, 511 (1980)

7. Novikov, S.P.: Magnetic Bloch functions and vector bundles. Sov. Math. Dokl. 23, 298 (1981)

8. Niu, Q., Thouless, D.J.: Quantised adiabatic charge transport in the presence of substrate disorder and many-body interaction. J. Phys. A 17, 2453 (1984)

9. Niu, Q., Thouless, D.J., Wu, Y.S.: Quantized Hall conductance as a topological invariant. Phys. Rev. B 31, 3372 (1985)

10. Avron, J.E., Seiler, R.: Quantization of the Hall conductance for general, multiparticle Schrödinger Hamiltonians. Phys. Rev. Lett. 54, 259 (1985)

11. Avron, J.E., Seiler, R., Shapiro, B.: Generic properties of quantum Hall Hamiltonians for finite systems. Nucl. Phys. B 265 [FS15], 364 (1986)

12. Tao, R., Haldane, F.D.M.: Impurity effect, degeneracy, and topological invariant in the quantum Hall effect. Phys. Rev. B 33, 3844 (1986)

13. Grümm, H.R., Narnhofer, H., Thirring, W.: On the Hall current in quantum theory. Acta Physica Austriaca 57, 175 (1985)

14. Kunz, H. Zumbach, G.: The static Hall conductivity in the presence of a strong magnetic field and random impurities, Nucl. Phys. B 270 [FS16], 347 (1986) 
15. Landau, L., Lifchitz, E.: Mécanique quantique - MIR (1966)

16. Delyon, F., Lévy, Y., Souillard, B.: Anderson localization for multi-dimensional systems at large disorder or large energy. Commun. Math. Phys. 100, 463 (1985)

17. Fröhlich, J., Martinelli, F., Scoppola, F., Spencer, T.: Constructive proof of localization in the Anderson tight binding model. Commun. Math. Phys. 101, 21 (1985)

18. Simon, B., Taylor, M., Wolff, T.: Some rigorous results for the Anderson model. Phys. Rev. Lett. 54, 1589 (1985)

19. Fröhlich, J., Spencer, T.: Absence of diffusion in the Anderson tight binding model for large disorder or low energy. Commun. Math. Phys. 88, 151 (1983)

20. Martinelli, F., Holden, H.: On absence of diffusion near the bottom of the spectrum for a random Schrödinger operator on $L^{2}\left(R^{v}\right)$. Commun. Math. Phys. 93, 197 (1984)

21. Aoki, H., Ando, T.: Critical localization in two-dimensional Landau quantization. Phys. Rev. Lett. 54, 831 (1985)

22. Weidmann, J.: Linear operators in Hilbert spaces. Berlin, Heidelberg, New York: Springer 1980

23. Thouless, D.J.: Localisation and the two-dimensional Hall effect. J. Phys. C 14, 3475 (1981)

24. Kato, T.: Perturbation theory for linear operators, Sect. 4. Berlin, Heidelberg, New York: Springer 1980

Communicated by E. Lieb

Received December 10, 1986 
